BNL 65907

\title{
LASER-ELECTRON COMPTON INTERACTION IN PLASMA CHANNELS
}

\author{
I.V. Pogorelsky, I Ben-Zvi \\ Brookhaven National Laboratory \\ Upton, New York, USA \\ T. Hirose \\ Physics Department \\ Tokyo Metropolitan University
}

October 1998

\author{
National Synchrotron Light Source \\ Brookhaven National Laboratory \\ Operated by \\ Brookhaven Science Associates \\ Upton, NY 11973 \\ Under Contract with the United States Department of Energy \\ Contract Number DE-AC02-98CH10886
}




\section{DISCLAIMER}

This report was prepared as an account of work sponsored by an agency of the United States Government. Neither the United States Government nor any agency thereof, nor any of their employees, nor any of their contractors, subcontractors or their employees, makes any warranty, express or implied, or assumes any legal liability or responsibility for the accuracy, completeness, or any third party's use or the results of such use of any information, apparatus, product, or process disclosed, or represents that its use would not infringe privately owned rights. Reference herein to any specific commercial product, process, or service by trade name, trademark, manufacturer, or otherwise, does not necessarily constitute or imply its endorsement, recommendation, or favoring by the United States Government or any agency thereof or its contractors or subcontractors. The views and opinions of authors expressed herein do not necessarily state or reflect those of the United States Government or any agency thereof 


\section{Laser-Electron Compton Interaction in Plasma Channels}

I.V. Pogorelsky*, I. Ben-Zvi

Accelerator Test Facility

National Synchrotron Light Source

Brookhaven National Laboratory

\section{T. Hirose}

Physics Department

Tokyo Metropolitan University

\section{Summary}

A concept of high intensity femtosecond laser synchrotron source (LSS) is based on Compton backscattering of focused electron and laser beams. The short Rayleigh length of the focused laser beam limits the length of interaction to a few picoseconds. However, the technology of the high repetition rate highaverage power picosecond lasers required for high put through LSS applications is not developed yet. Another problem associated with the picosecond laser pulses is undesirable nonlinear effects occurring when the laser photons are concentrated in a short time interval. To avoid the nonlinear Compton scattering, the laser beam has to be split, and the required hard radiation flux is accumulated over a number of consecutive interactions that complicates the LSS design.

In order to relieve the technological constraints and achieve a practically feasible high-power laser synchrotron source, we propose to confine the laser-electron interaction region in the extended plasma channel. This approach permits to use nanosecond laser pulses instead of the picosecond pulses. That helps to avoid the nonlinear Compton scattering regime and allows to utilize already existing technology of the high-repetition rate TEA $\mathrm{CO}_{2}$ lasers operating at the atmospheric pressure.

We demonstrate the advantages of the channeled LSS approach by the example of the prospective polarized positron source for Japan Linear Collider.

*Tel: (516) 344-5801, Fax: (516) 344-3115, E:mail: igor@bnl.gov 


\section{Introduction}

Development of high-brightness, quasi-monochromatic x-ray and $\gamma$-ray sources is a fast advancing area of scientific research. One of the most promising methods to generate the ultra-intense hard radiation flashes with a controlled polarization is based on interaction between pulsed laser beams with subpicosecond relativistic electron bunches. A laser interacting with a relativistic electron beam behaves like a wiggler in the conventional synchrotron light sources but of an extremely short period equal to half of the laser wavelength. This approach called laser synchrotron source (LSS) [1] opens a route to relatively compact, high-brightness femtosecond x-ray and gamma sources. Radiation generated by the LSS is characterized by high monochromaticity, brightness, efficiency, wavelength-tunability, polarization-control that are essential for the next generation high energy photon sources.

To achieve a high peak intensity and repetition rate, the LSS requires a high-power laser and a high brightness electron accelerator both operating at a high repetition rate. In addition, the interacting electron and laser beams need to be tightly focused. The short Rayleigh length of the focused laser beam limits the duration of the laser pulse to a few picoseconds. Contemporary linear accelerators with the photocathode electron injectors are well suited to the LSS. However, the technology of the high repetition rate highaverage power picosecond lasers is not developed yet. Another problem associated with the picosecond laser pulses is undesirable nonlinear Compton scattering occurring when the laser photons are concentrated in a short time interval. To avoid the nonlinear regime, the laser beam should be split, and the required gamma flux is accumulated over multiple consecutive interactions that significantly complicates the LSS design.

In order to abate the technological constraints and achieve a practically feasible high intensity LSS, we propose to confine the laser-electron interaction region in the extended plasma channel. This approach allows the use of nanosecond laser pulses instead of the picosecond pulses and relieves both mentioned above problems. In particular, it avoids the nonlinear Compton scattering regime and utilizes existant technology of highrepetition rate TEA (transverse electrical discharge, atmospheric pressure) $\mathrm{CO}_{2}$ lasers. High repetition rate, multi-kilowatt average power and a relatively short, $\sim 1 \mathrm{~ns}$, pulse duration have been demonstrated with such lasers [2]. This adds to other arguments that favor the long-wavelength $\mathrm{CO}_{2}$ laser as the LSS driver [3-5].

The proposed concept of using channeled $\mathrm{CO}_{2}$ laser beams may benefit a number of potential LSS applications in science and engineering. Among these applications is a novel method of generating the polarized positrons proposed for the Japan Linear Collider (JLC) $[6,7]$.

Polarization of electrons and positrons in future linear colliders will play an important role for experimental verification of the standard model and for a search of new phenomena beyond the standard model $[6,8]$. A prospective high-intensity polarized positron source (PPS) is based on production of the electron-positron pairs when the LSS (in other words, Compton scattered) polarized gamma-quanta are stopped at the foil target [6].

Observation of the first polarized positrons at KEK, Japan [9] proves the viability of this basic approach and the need for further development in this direction. This became a subject of a collaboration on the JLC PPS development recently established between KEK, Tokyo Metropolitan University (TMU) and Brookhaven National Laboratory (BNL) [10]. 
The concept of guiding high intensity pulsed laser beams in plasma channels has been conceived and experimentally demonstrated. However, the problem of producing the channeled high-repetition rate $\mathrm{CO}_{2}$ laser beams as well as their interaction with electron bunches has never been addressed and needs experimental verification. We propose that the KEK-TMU-BNL collaboration may be expanded to carry on research on the polarized Compton scattering in plasma channels.

The rest of the paper is organized as follows. In Section 2 we review fundamentals of the linear Compton scattering comparing the free space and channeled interaction regimes. Brief notes on principles and status of the short-pulse $\mathrm{CO}_{2}$ lasers and high-power laser channeling are given, correspondingly, in Sections 3 and 4. Section 5 suggests a modified design and format for JLC PPS that utilizes the plasma channeled high-power $\mathrm{CO}_{2}$ laser beams. A plan for proof-of-principle experiments at BNL and KEK is outlined in Section 6. 


\section{Compton scattering}

This section reviews the physical principles of the Compton scattering process in free space and inside the plasma channel.

A relativistic electron beam interacting with a counter-propagating laser beam emits radiation at a central wavelength

$$
\lambda_{X}=\lambda / 4 \gamma^{2}
$$

where $\gamma$ is the relativistic Lorentz factor and $\lambda$ is the laser wavelength.

The back-scattered photons are generated within a narrow cone with a solid angle of $\Omega=2 \pi \theta_{0}^{2}$, where

$$
\theta_{0}=1 / \gamma
$$

For the back-scattering geometry, the x-ray pulse length is defined primarily by the electron bunch duration:

$$
\tau_{X} \approx \tau_{b}+\frac{\tau_{L}}{4 \gamma^{2}}
$$

and for a practically meaningful case when $\tau_{L} \ll 4 \gamma^{2} \tau_{b}$ we can consider $\tau_{X} \approx \tau_{b}$. It follows that relatively long laser pulses may be tolerated without a noticeable increase in the x-ray pulse duration above $\tau_{b}$.

The number of x-ray photons, $N_{X}$, is given by

$$
N_{X} \approx \frac{8 \mathbf{E}_{L} Q r_{e}^{2} \lambda}{3 h e r_{L}^{2}}
$$

where $\mathbf{E}_{L}$ is the laser pulse energy and $Q$ is the electron bunch charge.

Eq.(4) for the x-ray flux is derived under the assumption that the laser beam is focused to $r_{L} \geq r_{b}$ and that the electron/photon interaction length extends over the overlap distance defined by the electron and laser pulse duration,

$$
L_{L} \approx c\left(\tau_{L}+\tau_{b}\right) / 2 .
$$

The low emittance permits tight transverse confinement of the relativistic electron beam over extended distances. Due to diffraction, when focused to the same spot, the laser beam diverges much faster. Thus, the interaction region (overlap of waists) of counterpropagating focused electron and laser beams is defined primarily by the laser waist length:

$$
L_{L} \approx \pi^{2} r_{L}^{2} / \lambda
$$

Assuming $\tau_{L} \gg \tau_{b}$ Eqs. (5) and (6) can be reduced to

$$
\frac{\tau_{L} \lambda}{r_{L}^{2}} \approx \frac{2 \pi^{2}}{c} \text {. }
$$

Thus, in order to reach high $N_{X}$ value, the reduction in $r_{L}$ is necessary. This may be achieved through a reduction of $\tau_{L}$. However, following this trend, we soon enter a nonlinear regime when the normalized laser strength $a>1$, where

$$
a=4.5 \times \frac{\lambda}{r_{L}} \times\left(\frac{\mathbf{E}_{L}[J]}{\tau_{L}[p s]}\right)^{1 / 2} .
$$

The problem associated with the nonlinear regime is a loss of monochromaticity of the produced Compton quanta which will be scattered over many harmonics of the 
fundamental wavelength defined by Eq. (1). There are also serious technological problems when we try to deliver a high laser energy in a short pulse. We will talk about this in the next section. Thus, the free space linear Compton interaction presents certain restrictions for selecting the laser power, pulse duration, and the focal spot size.

The idea of plasma channeling of the laser beam breaks the fine restriction imposed by Eq. (7) thus opening new possibilities to increase $N_{X}$. Due to the confinement of the laser beam over a distance much longer than the Rayleigh length, the laser pulse length can be increased without the proportional increase in $r_{L}^{2}$ called for in Eq. (7) . For the higher energy and proportionally longer laser pulses, the increased number of laser photons interacting with electrons results in higher $N_{X}$ without entering the nonlinear regime.

The above statement is illustrated by examples in Table 1 where various regimes of the Compton interaction are considered using the $30 \mathrm{~J}$ laser pulses. We observe that channeling of the laser beam permits efficient utilization of the relatively long highenergy laser pulses. With shorter laser pulses of the same energy we can not avoid the nonlinear regime unless the laser focus spot is expanded with the consecutive reduction in $N_{X}$. 


\section{Short pulse $\mathrm{CO}_{2}$ laser technology}

From Eq.(4), we see that the number of generated $\mathrm{x}$-ray photons is proportional to $\lambda$. This stems from the fact that the number of photons delivered within the laser pulse is inversely proportional to the photon energy. This justifies use of the long-wavelength $\mathrm{CO}_{2}$ laser for intense $\mathrm{x}$-ray and gamma generation. An additional reason is due to the potentials of the $\mathrm{CO}_{2}$ laser technology for the high repetition rates and high average power $[2,11]$.

An important laser parameter to consider is the pulse duration. As we have seen in the previous paragraph, the picosecond laser pulses are required to achieve a high efficiency of the Compton interaction in the free space. The gain spectral bandwidth is a prime physical parameter that enables generation and amplification of picosecond laser pulses. The spectral gain in the molecular gas discharge is periodically modulated by the rotational structure. Pressure broadening of the $\mathrm{CO}_{2}$ gain spectrum at $\sim 10$ atm into a 1 $\mathrm{THz}$ quasi-continuum permits amplification of the 1-ps $\mathrm{CO}_{2}$ laser beams [12].

To attain an energy of several joules in a picosecond pulse (that is equivalent to terawatt peak power), a $\sim 10$-atm, $\sim 10-l \mathrm{CO}_{2}$ amplifier is required. To pump such an amplifier, a multi-kJ, sub-microsecond, 1-MV electrical pulse shall be applied to the x-ray preionized discharge. This presents a significant technological problem which has been actually solved at the BNL-ATF where the first laser with such parameters is close to completion [13].

As is discussed in Section 2, nanosecond pulses can be tolerated if the concept of laser channeling is adopted. A relatively low, down to $1 \mathrm{~atm}$, gas pressure is sufficient to generate and amplify $\sim 1 \mathrm{~ns} \mathrm{CO}_{2}$ laser pulses. The low pressure relieves a number of technical problems associated with the high-pressure lasers. These problems and their solutions are listed as follows:

First, proportional to gas pressure reduction in the discharge voltage and current permits the use of existing high-power electrical modulators capable to pump the TEA $\mathrm{CO}_{2}$ lasers at the $\mathrm{kHz}$ repetition rate. The corresponding problem is not solved for the 1-MV lowinductance generators required for large 10-atm lasers.

Second, low or no mechanical stress to the output window typical for the 1-atm laser amplifier provides a simple solution (in terms of the window size and thickness) to the high laser energy extraction that is one of the limiting factors for the high-pressure laser technology.

Third, the low pressure allows to use the UV preionization employed inside the gas volume and capable of the $\mathrm{kHz}$ repetition rate. Alternatively, the $\mathrm{x}$-ray preionizer used in high-pressure lasers requires pumping to a deep vacuum after each shot that restricts the discharge repetition rate.

Finally, the high average power in the $\mathrm{CO}_{2}$ amplifier is possible due to heat removal via fast gas exchange. High-productivity turbines and fans are available for the $1 \mathrm{~atm}$ and lower gas pressure. On the other hand, fast gas circulation is considerably more difficult for operation in a compressed gas.

Based on these technical considerations, we propose the LSS design based on plasma channeled nanosecond laser pulses produced by TEA $\mathrm{CO}_{2}$ lasers which are capable of several $\mathrm{kW}$ average power and $\sim 10 \%$ wall-plug efficiency. 


\section{Plasma channel for $\mathrm{CO}_{2}$ laser guiding}

As seen in the above discussion, the plasma channel relaxes challenges for efficient, high repetition rate Compton production of $\mathrm{x}$ - and $\gamma$-rays. However, we encounter a new problem of producing laser channels. Fortunately, the problem of the laser channel formation looks feasible.

There are a number of proposed schemes and experimental demonstrations for channeling of high intensity laser pulses. They include laser guiding in microcapillary tubes [14], or in plasma channels produced by electric discharge in the dielectric capillary tube [15], and by laser breakdown in the gas [16, 17].

All the plasma channeling experiments have been performed using picosecond solid state lasers. However, as shown in the Appendix, guiding in a plasma channel is not sensitive to the laser wavelength. That means that the $\mathrm{CO}_{2}$ laser may perform as well as a solid state laser that has 10 times smaller wavelength. It is confirmed by simulations [18] that agree with the experimental results obtained with a solid state laser [15] and extend predictions to the $10-\mu \mathrm{m}$ laser case.

For the LSS design we propose the study of several most promising options of channeling the laser pulse. The plasma channel research has significance to other applications as well, including plasma wakefield acceleration. 


\section{$5 \quad$ Plasma channeled $\gamma$-source for JLC}

The JLC project requires $\sim 1.2 \mathrm{kHz}$ cumulative repetition rate of the delivered positron bunches. Thus, a polarized gamma source shall be of the correspondingly high repetition rate. To obtain such a cumulative repetition rate, the adopted plan is to deliver the electron and laser pulses in trains of 85 pulses with the $2.8 \mathrm{~ns}$ separation between the pulses and $150 \mathrm{~Hz}$ repetition rate between the trains [19]. A feasibility of such $\mathrm{CO}_{2}$ laser regime was first suggested in [20]. The current PPC conceptual design assumes the matched configuration when the electron and laser beams are both focused to the $30 \mu \mathrm{m}$ spot and pulse duration is accordingly small, $10 \mathrm{ps}$ [19].

Using a plasma channel of the same $30 \mu \mathrm{m}$ radius introduces a possibility to choose a longer laser pulse duration and correspondingly higher laser pulse energy without entering the nonlinear regime. Note that this modification does not reduce the cumulative laser output power to drive the JLC PPS, that is $\sim 120 \mathrm{~kW}$. However, plasma channeling permits to reduce the number of the required laser sources to compare with the adopted PPS design [19] if the average power of each laser is higher than $3 \mathrm{~kW}$.

For a given average power, the laser repetition rate is inversely proportional to the energy per pulse. This permits a more practical regime for the $\mathrm{CO}_{2}$ laser operation. For example, we can talk about bursts of 8 pulses of the $10 \mathrm{~J}$ energy each spaced at $28 \mathrm{~ns}$, instead of 85 pulses of the $250 \mathrm{~mJ}$ energy at the $2.8 \mathrm{~ns}$ period according to the current JLC PPS design. Then, each electron microbunch $(6 \mathrm{nC})$ interacts with a single laser pulse to acquire $5 \times 10^{11}$ gamma quanta. The proposed modified format for the electron/laser interaction is illustrated by Fig.1. If we assume the average laser power at $3 \mathrm{~kW}$, a possible pulse format is when the laser energy is 2.5-3.0 J per pulse and every electron bunch needs to interact with four laser pulses. This configuration is compared in Table 2 with the freespace interaction regime.

The total length occupied by the consecutive channels is limited by the angular divergence of the electron beam and the produced Compton radiation. The practical limit for the length of the JLC PPS Compton source is $2 \mathrm{~m}$. The limited number of channels requires a method of fitting several laser beams into a single channel. One of the possibilities is to use several laser beams incident to the parabolic mirror at slightly different angles and fit them into the channel with the sufficiently wide conical opening. For the $1 \mathrm{~ns}$ laser pulse, a $15 \mathrm{~cm}$ long plasma channel is sufficient. Interacting with laser 4 times in 4 channels (every mirror combines 10 laser beams) each electron bunch produces the same number of gamma photons as previously after 40 interactions with the 10 times less energetic laser pulses.

The use of plasma channels and the above mentioned modification of the laser parameters simplifies the laser design considerably. First, the 8-micropulse burst with the $28 \mathrm{~ns}$ period may be naturally produced from the regenerative preamplifier with the controlled electro-optical switch.

Then, a plasma channel permits longer laser pulses and, thus, lower pressure. For example, 1-atm lasers can be used. The low pressure facilitates maintaining a high repetition rate discharge. In addition, the gain lifetime is longer proportionally to the pressure reduction that may help to arrange the multi-pass or regenerative amplification of the pulse burst over the $\sim 250$ ns time span.

A principal diagram illustrating the optical configuration of a feasible $\mathrm{CO}_{2}$ laser to drive the $\gamma$-source for JLC is shown in Fig.2. The laser chain starts with the $\sim 100$-ns pulse generated by the conventional $\mathrm{CO}_{2}$ laser, its gating to $1 \mathrm{~ns}$ by a fast electro-optical switch, and amplification in a regenerative and then multi-pass regime. 
Multi-bunch energy extraction in the train of a total duration of $\sim 250 \mathrm{~ns}$ is an efficient regime for the atmospheric $\mathrm{CO}_{2}$ laser. Indeed, the rotational relaxation time $\sim 4$ ns and the $\mathrm{N}_{2}(\mathrm{v}=1) \Rightarrow \mathrm{CO}_{2}(001)$ energy transfer time $100 \mathrm{~ns}$ (both at 1 atm) permit to extract efficiently the energy stored not just at the $\mathrm{CO}_{2}(001)$ vibrational mode but from the $\mathrm{N}_{2}(\mathrm{v}=1)$ energy reservoir as well. Although this regime was not attempted so far, there is an expectation that the wall-plug efficiency of such a laser will approach 5-10\% which is a routine for conventional $\mathrm{CO}_{2}$ lasers. 


\section{Proof-of-Principle Experiments at BNL and KEK}

For a success of the proposed LSS scheme it is essential to achieve tight focusing and fine alignment of the interacting electron and laser beams. Accordingly, the experiment setup shall include the following prime components:

- An interaction vacuum-chamber with the optics assembly for focusing and alignment of the laser beam, assembly for the plasma channel production, and positioning diagnostics for the laser and electron beams.

- Focusing and steering electromagnets for the e-beam.

- X-ray diagnostics.

The preferred location of the Compton experiment is at the end of the ATF beam-line \#1. The tight focusing of the electron beam will be established in this position by the quadrupole triplet. Placement of the Compton interaction cell before the bending dipole magnet at the end of beam-line \#1 permits $\mathrm{x}$-ray separation from the spent e-beam and the $\mathrm{x}$-ray extraction to diagnostics through the Be foil window.

The Compton interaction vacuum cell, shown in Fig.3, will accommodate the following primary components:

a) Optics that telescope and redirect the sidewise incident $\mathrm{CO}_{2}$ laser beam, focus it at the electron-beam axis at the channel entrance and extract the spent laser.

b) Provisions to produce the plasma channel inside the Compton cell.

c) Two pop-in e-beam position and size monitors that could be remotely inserted upstream and downstream of the channel to control the electron-beam size, position, and direction.

$5 \mathrm{~mm}$ diameter holes are drilled through the parabolic mirrors in order to transmit the ebeam and the produced x-rays. To avoid the loss of laser energy and plasma ignition at the edges of the hole, a "donut"-shaped laser beam (having zero intensity at the center) will be used. Such laser beam profile may be formed using an axicon telescope prior to delivery of the laser beam into the cell.

The experiment will be equipped with the x-ray diagnostics (not shown in Fig.3). Its complexity and capabilities will depend upon funding. In the case of a tight budget, we may be limited with the $\mathrm{x}$-ray dose detector which still permits to satisfy the principal goal of the experiment - to demonstrate the high efficiency $\mathrm{x}$-ray production and to optimize the laser/electron interaction geometry.

When possible, more advanced characterization of the x-ray beams may be implemented that permits measurements of temporal, spectral, spatial, and angular parameters. For example, the spectral selectivity will be introduced by the crystal (graphite) monochromator. The most reliable way to measure the $\mathrm{x}$-ray pulse duration is by the $\mathrm{x}$ ray streak-camera, with the subpicosecond time resolution, which is commercially available.

At the KEK-ATF, generation of polarized positrons with the plasma-channeled LSS will be investigated in terms of polarization state, production rate, angular spread of generated positrons etc. The results will be compared with the experiment on free-space Compton scattering which is now under progress at KEK.

The initial stages of the project will utilize the presently available electron beam and $\mathrm{CO}_{2}$ laser at the BNL Accelerator Test Facility (ATF) and the damping ring at the KEK-ATF. A newly developed plasma channel experimental set-up will be installed into the electron beamline for Compton scattering experiments. The BNL-ATF $\mathrm{CO}_{2}$ laser will be modified 
to fit better to the PPS requirements until a new $\mathrm{CO}_{2}$ laser will be assembled at the KEKATF. This schedule permits to utilize in the full manner the scientific and technical potential of both facilities, to initiate promptly proof-of-principle experiments using the existing laser and the $50 \mathrm{MeV}$ electron linac at the BNL-ATF, and to use the acquired experience later for the verification of the original idea [6] by observing polarized positron production when the KEK-ATF laser will be available for prototype experiments with the $1.56 \mathrm{GeV}$ KEK electron beam. 


\section{References}

1. P. Spranle, A. Ting, E. Esarey, A. Fisher, J. Appl. Phys., 72, 5032 (1992)

2. "Handbook of Molecular Lasers", ed. P.K. Cheo, Marcel Dekker, Inc, New-York, 1987

3. I.V. Pogorelsky, "High-Intensity Laser Synchrotron x-Ray Source", BNL Preprint, BNL62447 (1995)

4. I.V. Pogorelsky, "Ultra-Bright x-Ray and Gamma Sources by Compton Backscattering of $\mathrm{CO}_{2}$ Laser Beams", Nucl. Instrum. And Methods in Phys. Res. A 411, 172-187 (1998)

5. I.V.Pogorelsky, I. Ben-Zvi, "Emerging terawatt picosecond $\mathrm{CO}_{2}$ laser technology and possible applications in accelerator physics", Particle Accel. Conf. 97, Vancouver, B.C., Canada, May 12-16, 1997, http://www.triumf.ca/pac97/papers/pdf/6V040.PDF

6. T. Hirose, "Important Role of Polarized Positron Beams in Future Linear Colliders", Proc. Of Intern. Workshop on Physics and Experiments with Linear Colliders, September 1995, Morioka Iwate Japan, September 8-12, 1995, Word Science, Singapore, 748 (1996);

7. T. Okugi, Y. Kurihara, M. Chiba, A. Endo, R. Hamatsu, T. Hirose, T. Kumita, T. Omori, Y. Takeuchi, and M. Yosioka, "Proposed Method to Produce a Highly Polarized e ${ }^{+}$Beam for Linear Colliders", Jpn. J. Appl. Phys., 35, 3677 (1996)

8. T. Omori, Y. Kurihara, Y. Takeuchi, A. Endo, A. Tsunemi, T. Hirose, T. Kumita, T. Okugi, M. Chiba, R. Hamatsu, and H. Ishiyama, "Polarized Posotron Source for Linear Colliders", Proc. of Intern. Workshop on New Kinds of Positron Sources for Linear Colliders, March 47, 1997 at SLAC, 341, SLAC-R-502

9. T.Hirose,"Generation of polarized positrons via laser-Compton scattering at the KEK damping ring" Proceedings of the First Asian Particle Accelerator Conference, APAC98, March 23-27, 1998 at KEK, Tsukuba, Japan

10. "Study of Compton Scattering of Picosecond Electron and $\mathrm{CO}_{2}$ Laser Beams to Prototype the Polarized Positron Source for Japan Linear Collider", Proposal for an experiment at the Brookhaven Accelerator Test Facility submitted by TMU, KEK, SUNY Stony Brook, and NSLS/ATF, March 30, 1998, http://www.nsls.bnl.gov/AccTest/experiments/Compton/pro.htm

11. I.V.Pogorelsky, A. van Steenbergen, R. Fernow, W.D. Kimura, S.V. Bulanov, " $\mathrm{CO}_{2}$ Laser Technology for Advanced Particle Accelerators", 7th Workshop on Advanced Accelerator Concepts, October 12-18, 1996, Lake Tahoe, CA, AIP Conference Proceedings 398, 923 (1997)

12. A.J. Alcock and P.B. Corkum, "Ultra-Short Pulse Generation with $\mathrm{CO}_{2}$ Lasers", Phil. Trans. Royal Soc. London., A298, 365 (1980)

13. I.V.Pogorelsky, I. Ben-Zvi, J. Skaritka, Z. Segalov, M. Babzien, K. Kusche, I.K. Meshkovsky, V.A. Lekomtsev, A.A. Dublov, Yu.A. Boloshin, and G.A. Baranov, "The First Terawatt Picosecond $\mathrm{CO}_{2}$ Laser for Advanced Accelerator Studies at the Brookhaven ATF", 7th Workshop on Advanced Accelerator Concepts, October 12-18, 1996, Lake Tahoe, CA, AIP Conference Proceedings 398, 937 (1997)

14. S. Jackel, R. Burris, J. Grun, A. Ting, C. Manka, K. Evans, and J. Kosakovskii, "Channeling of Terawatt Laser Pulses by Use of Hollow Waveguides", Opt. Lett. 20, 1086-1088 (1995) 
15. Y. Ehrlich, C. Cohen, A. Zigler, J. Krall, P. Sprangle, and E. Esarey, "Guiding of High Intensity Laser Pulses in Straight and Curved Plasma Channel Experiments", Phys. Rev. Lett. 77, 4186-4189 (1996)

16. C.D. Durfee III \& H.M. Milchberg, Phys. Rev. Lett., 71, 2409 (1993)

17. I.V. Pogorelsky, W.D. Kimura, and Y. Liu, "Waveguiding by Axicon-Focused Laser Beams", 6th Workshop on Advanced Accelerator Concepts, Jun. 12-18, 1994, Lake Geneva, WI, AIP, 335, 419 (1995)

18. N.A. Bobrova, S.V. Bulanov, D. Farina, R. Pozzoli, T.L. Razinkova, and P.V. Sasorov, "Wave Guiding of the Intense Ultrashort Laser Pulses in Capillary Plasmas", published in Journal of Physical Society of Japan (1998)

19. A. Tsunemi, A. Endo, M. Washio, T. Hirose, Y. Kurihara, T. Omori, and J. Urakawa, Picosecond $\mathrm{CO}_{2}$ Laser Driven Polarized Positron Source for Japan Linear Collider" at Lasers '97, December 1997, New Orleans, Louisiana SRS Press, McLean, VA, $839-844$ (1998)

20. I.V. Pogorelsky, "Terawatt $\mathrm{CO}_{2}$ Laser Technology for Strong Field Physics Applications", $I^{\text {st }}$ Conf. On Superstrong Fields in Plasmas, Varenna, Italy, August 27 - September 2, 1997, AIP Conf. Proc., 426, 415 (1998) 


\section{Figures}

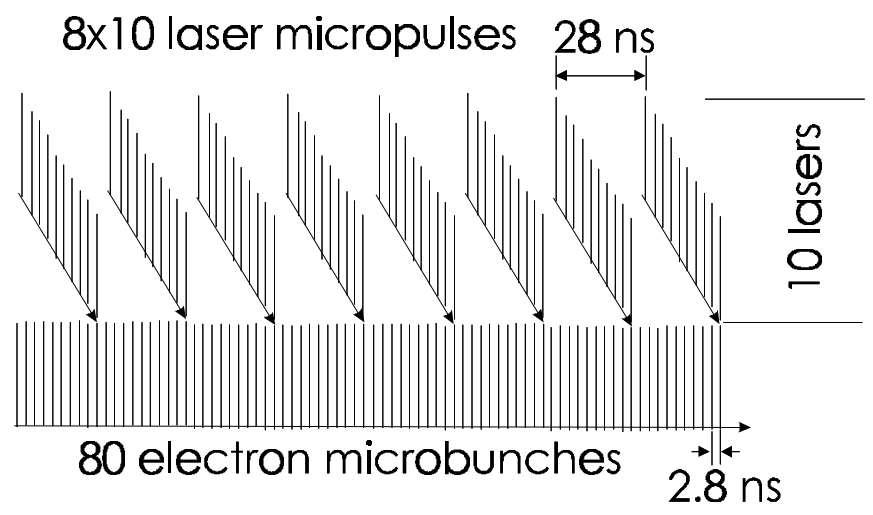

Fig.1 Electron-laser interaction sequence. This example shows how ten lasers can be combined to interact with 80 bunches. It is assumed that each laser pulse has $10 \mathrm{~J}$ energy.

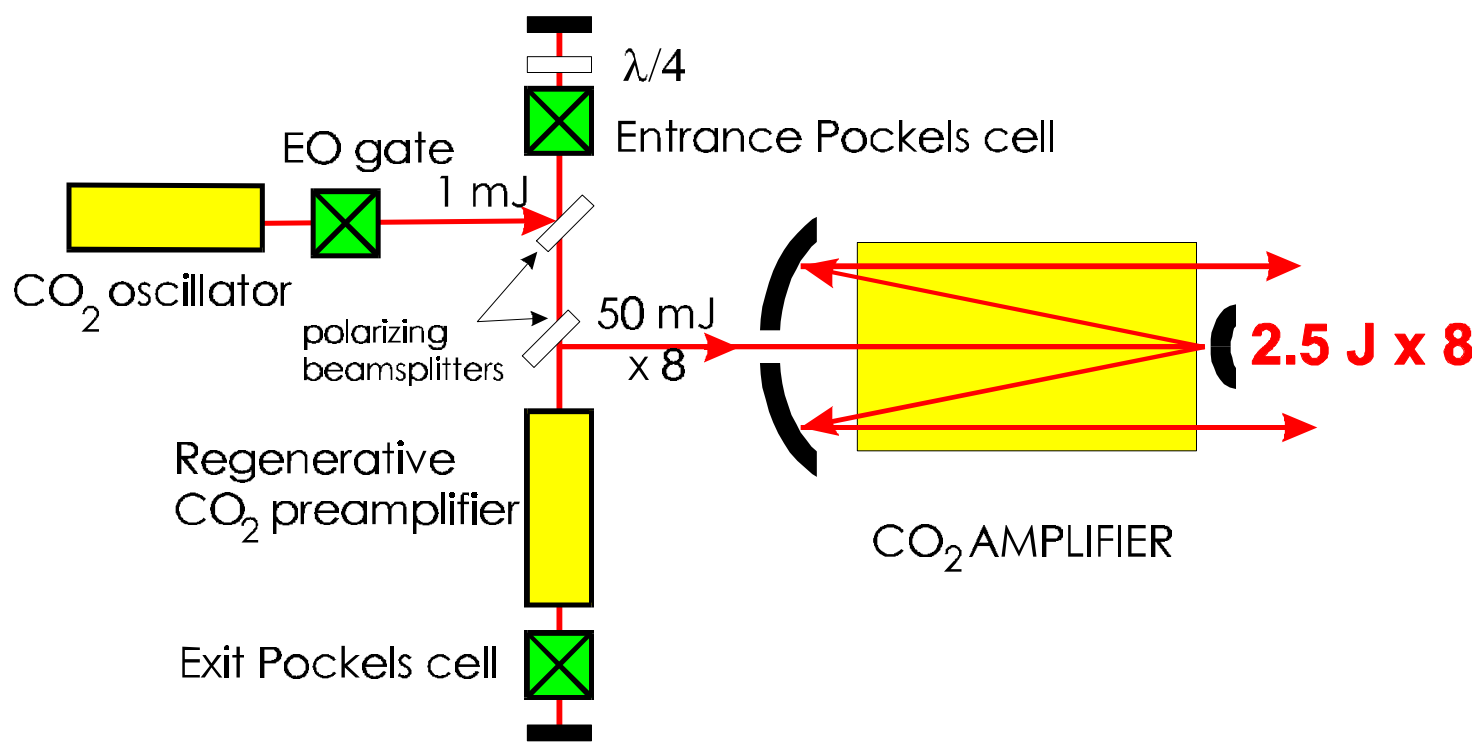

Fig.2 Principle optical setup of the terawatt picosecond $\mathrm{CO}_{2}$ laser system with the multi-pulse train capability 


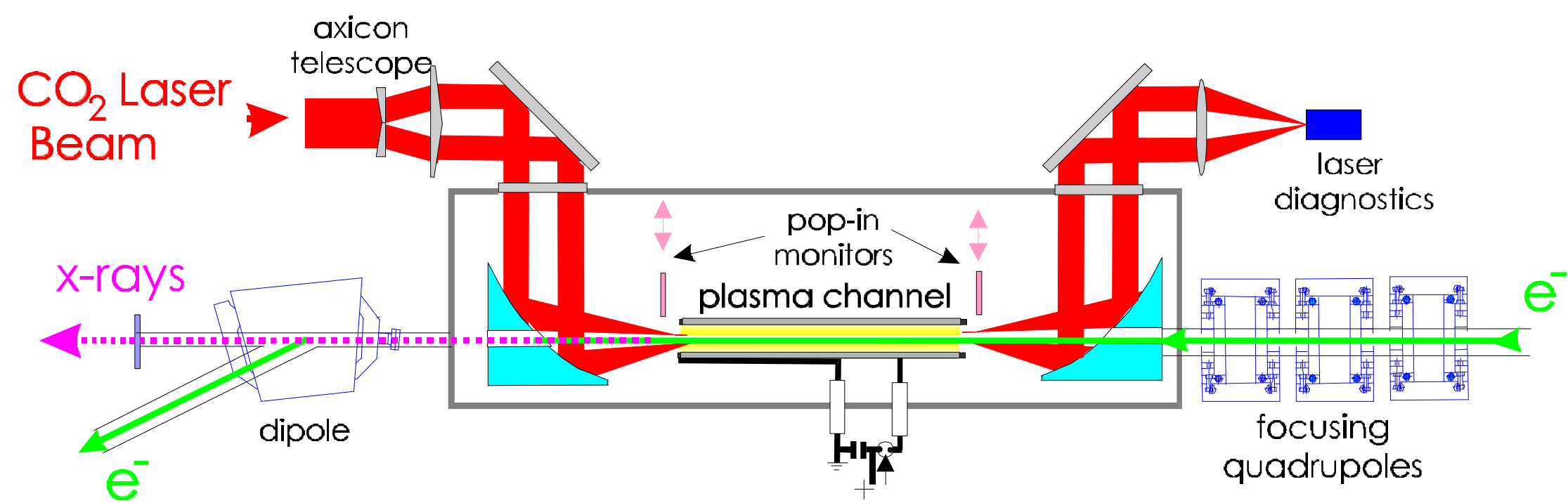

Fig.3 Design of the Compton scattering experiment in the plasma channel. (Discharge in the capillary tube is shown as an example. Other methods of channel formation are under consideration as well.) 


\section{TABLES}

Table 1 Compton X-Ray Source Design Parameters

\begin{tabular}{|c|c|c|c|}
\hline Interaction Conditions: & $\begin{array}{l}0.3-\mathrm{TW}, 100-\mathrm{ps} \\
\text { free space }\end{array}$ & $\begin{array}{l}\text { 6-TW, 5-ps } \\
\text { free space }\end{array}$ & $\begin{array}{l}\text { 0.3-TW, 100-ps } \\
\text { channeled }\end{array}$ \\
\hline \multicolumn{4}{|c|}{$\mathrm{CO}_{2}$ LASER } \\
\hline Pulse Duration [ps] & 100 & 5 & 100 \\
\hline Peak Power [TW] & 0.3 & 6 & 0.3 \\
\hline Radius at Focus $[\mu \mathrm{m}]$ & 100 & 30 & 30 \\
\hline Normalized laser strength & 0.25 & 3.7 & 0.8 \\
\hline Waist (or Channel) Length [mm] & 9 (waist) & 1.2 (waist) & 20 (channel) \\
\hline \multicolumn{4}{|c|}{ ELECTRON BEAM } \\
\hline Energy $[\mathrm{MeV}]$ & 70 & 70 & 70 \\
\hline Bunch Charge [nC] & 0.5 & 0.5 & 0.5 \\
\hline Bunch Duration FWHM [ps] & 3 & 3 & 3 \\
\hline Normalized Emittance [mm.mrad] & 2 & 2 & 2 \\
\hline Radius at Focus $[\mu \mathrm{m}]$ & 140 & 40 & 40 \\
\hline Waist Length $[\mathrm{cm}]$ & 1000 & 80 & 80 \\
\hline Electron Momentum Spread & $0.05 \%$ & $0.05 \%$ & $0.05 \%$ \\
\hline \multicolumn{4}{|c|}{ X RAYS } \\
\hline Wavelength $[\AA]$ & 1.3 & $1.3+$ harmonics & 1.3 \\
\hline Pulse Duration [ps] & 3 & 3 & 3 \\
\hline Angular Spread [mrad] & 7 & 7 & 7 \\
\hline Spectral Bandwidth & $0.12 \%$ & $1.5 \%$ & $1 \%$ \\
\hline Photons per Pulse & $5 \times 10^{9}$ & $10^{11}$ & $10^{11}$ \\
\hline
\end{tabular}

Table 2 Laser Drivers and Interaction Geometry for JLC Polarized Positron Source

\begin{tabular}{||l|c|c|}
\hline \multicolumn{1}{|c|}{ Parameter } & Current Design & Proposed New Design \\
\hline \multicolumn{3}{|c|}{ CO $_{\mathbf{2}}$ LASER } \\
\hline Microbunch Duration $[\mathrm{ps}]$ & $\mathbf{1 0}$ & $\mathbf{1 0 0 0}$ \\
\hline Microbunch Energy $[\mathrm{J}]$ & $\mathbf{0 . 2 5}$ & $\mathbf{2 . 5}$ \\
\hline Peak Power $[\mathrm{GW}]$ & $\mathbf{2 5}$ & $\mathbf{2 . 5}$ \\
\hline Microbunch period $[\mathrm{ns}]$ & $\mathbf{2 . 8}$ & $\mathbf{2 8}$ \\
\hline Microbunch number & $\mathbf{8 5}$ & $\mathbf{8}$ \\
\hline Macrobunch Rep. Rate $[\mathrm{Hz}]$ & $\mathbf{1 5 0}$ & $\mathbf{1 5 0}$ \\
\hline Average Power $[\mathrm{kW}]$ & $\mathbf{3}$ & $\mathbf{1}$ \\
\hline Gas Pressure $[\mathrm{atm}]$ & $\mathbf{1 0}$ & \\
\hline
\end{tabular}




\begin{tabular}{|c|c|c|}
\hline Technological Challenges & $\begin{array}{l}\text { High-pressure, high rep. rate } \\
\text { lasers do not exist, multipulse } \\
\text { slicing and amplification never } \\
\text { demonstrated. } \quad \text { Requires } \\
\text { extensive R\&D. }\end{array}$ & $\begin{array}{l}\text { 1-atm, } \mathrm{kW}, \mathrm{kHz} \mathrm{CO}_{2} \text { lasers } \\
\text { exist, multiple pulses can be } \\
\text { produced from regenerative } \\
\text { amplifier. Requires moderate } \\
\text { R\&D. }\end{array}$ \\
\hline \multicolumn{3}{|c|}{ INTERACTION GEOMETRY } \\
\hline Number of lasers & 40 & 40 \\
\hline Number of mirrors & 40 & 4 \\
\hline Laser pulses per electron bunch & 40 & 4 \\
\hline Rotation Speed [MHz] & Stationary & 44.6 \\
\hline Interaction & Free space & Channel \\
\hline Radius at Focus $[\mu \mathrm{m}]$ & 30 & 30 \\
\hline Waist (or Channel) Length [mm] & 9 (waist) & 150 (channel) \\
\hline Total interaction length $[\mathrm{m}]$ & $2+?$ & 1.2 \\
\hline Technological Challenges & No major & $\begin{array}{l}\text { Requires } \mathrm{R} \& \mathrm{D} \text { in plasma } \\
\text { channeling of high power } \mathrm{CO}_{2} \\
\text { laser. }\end{array}$ \\
\hline
\end{tabular}

\section{APPENDIX}

\section{OPTICAL GUIDING IN PLASMA CHANNELS*}

In a plasma, the refractive index, $\eta$, depends upon $n_{e}$ and $\lambda$ according to the equation

$$
\eta=\eta_{0} \sqrt{1-\frac{e^{2} \lambda^{2} n_{e}}{\pi c^{2} m}}=\eta_{0} \sqrt{1-\frac{n_{e}}{n_{c r}}},
$$

where $n_{c r}=\pi m c^{2} / e^{2} \lambda^{2}$, known as the critical electron density. For $n_{e}<<n_{c r}$, Eq.(A1) takes the form

$$
\eta \approx \eta_{0}\left(1-\frac{n_{e}}{2 n_{c r}}\right) .
$$

The idea of a preformed plasma waveguide is based on changing the refractive index in a medium due to a developed plasma density gradient according to the equation

$$
\frac{\Delta \eta}{\eta_{0}}=-\frac{\Delta n_{e}}{2 n_{c r}}
$$

For an EM wave incident onto the plasma layer at the angle $\beta_{0}$ the condition for reflection is according to Snell's law

$$
\eta(r) \leq \eta_{0} \sin \beta_{0},
$$

or for oblique incidence $\quad \eta(r)-\eta_{0} \equiv \Delta \eta(r) \leq-\eta_{0} \theta_{0}^{2} / 2, \quad$ where $\theta_{0} \equiv 90^{0}-\beta_{0}, \quad$ and by Eq.(A3)

$$
\Delta n_{e} \geq n_{c r} \theta_{0}^{2} \text {. }
$$

If we consider a cylindrical plasma layer with the "wall height" to satisfy Eq.(A5), then for a focused Gaussian beam having a diffraction divergence

$$
\theta_{0}=\frac{\lambda}{r_{L} \pi}
$$

the condition of optical guiding inside a plasma cylinder is 


$$
\Delta n_{e} \geq \frac{m c^{2}}{e^{2} r_{L} \pi} \equiv\left(r_{e} r_{L}^{2} \pi\right)^{-1},
$$

where $r_{e}=2.82 \times 10^{-13} \mathrm{~cm}$ is the classic electron radius, or

$$
\Delta n_{e}\left[\mathrm{~cm}^{-3}\right]=1.13 \times 10^{20} / r_{L}^{2}[\mu \mathrm{m}] .
$$

Note, that the waveguiding condition, Eq.(A7), does not depend upon the laser wavelength.

* I. Pogorelsky, "Relativisticallty Strong $\mathrm{CO}_{2}$ Laser Driver for Plasma-Channeled Particle Acceleration”, Conference Lasers'95, December 4-8, 1995, Charleston, SC, STS Press, 104-111 (1996) 